\title{
Missing-Linker 2D Conductive Metal Organic Frameworks for Rapid Gas Detection
}

\author{
Congyue $L i u^{1}$, Yuming $G^{2}$, Cheng $L i u^{1}$, Shengtang $L i u^{1}$, Xiaoshan $L i^{1}$, Jing $M a^{2}$ and Mengning Ding ${ }^{1 *}$ \\ 1 Key Laboratory of Mesoscopic Chemistry, School of Chemistry and Chemical Engineering, Nanjing \\ University, Nanjing 210023, China \\ 2 Jiangsu Key Laboratory of Advanced Organic Materials, School of Chemistry and Chemical \\ Engineering, Nanjing University, Nanjing 210093, China
}

\section{Table of Contents:}

Figure S1. Characterization of oxidized HAB and HAB.

Figure S2. Synthesis of missing-linker amorphous Ni-HAB (aNi-HAB) and sensory device fabrication.

Figure S3. Scanning Electron Microscopy (SEM), X-ray Photoelectron Spectroscopy (XPS) of aNi-HAB. Fourier Transform Infrared Spectroscopy (FT-IR) of aNi-HAB and cNi-HAB.

Figure S4. Thermogravimetric Analysis (TGA), $\mathrm{N}_{2}$ adsorption-desorption isotherms BET and I-V curves of aNi-HAB.

Figure S5. Humidity sensor performance of crystal Ni-HAB (c-Ni-HAB).

Figure S6. SEM and Energy Dispersive X-ray Spectroscopy (EDS) of aMOF with four different metal centers.

Figure S7. FT-IR Spectrum of aMOF with four different metal centers.

Figure S8. DFT calculated band structure and density of states of cNi-HAB and aNi-HAB. 


\section{Methods}

Materials: All reagents and solvents were purchased from commercial supplier and used as received, without further purification. Hexaaminobenzene Trihydrochloride (97\%) were purchased from Alfa Aesar and Toronto Research Chemicals (TRC). Silicon wafers with $270 \mathrm{~nm}$ thick $\mathrm{SiO}_{2}$ grown thermally was purchased from SPI technologies.

Synthesis of missing-linker M-HAB (aNi-HAB, aCu-HAB, aFe-HAB, aCo-HAB). The as-purchased hexaaminobenzene (HAB) was kept in air in a $30{ }^{\circ} \mathrm{C}$ oven for 10 days to obtain oxidized ligands. Metal salt $\left(\mathrm{NiCl}_{2} \cdot 6 \mathrm{H}_{2} \mathrm{O}, \mathrm{CuCl}_{2} \cdot 2 \mathrm{H}_{2} \mathrm{O}, \mathrm{Fe}\left(\mathrm{NO}_{3}\right)_{3} \cdot 9 \mathrm{H}_{2} \mathrm{O}\right.$, and $\left.\mathrm{Co}\left(\mathrm{NO}_{3}\right)_{2} \cdot 6 \mathrm{H}_{2} \mathrm{O}\right)(0.072 \mathrm{mmol})$, triethylamine (4 vol\% aqueous solution, $\left.5 \mathrm{~mL}\right)$ and oxidized HAB $(0.035 \mathrm{mmol}$ aqueous solution, $5 \mathrm{~mL})$ were then mixed together in a 20 $\mathrm{mL}$ scintillation vial without stirring for $12 \mathrm{~h}$. Black precipitate was instantaneously produced. The prepared mixture was centrifuged, decanted and washed with DI water and then methanol for three times. The solid product was first dried at room temperature under nitrogen protection, and then activated in vacuum at $100{ }^{\circ} \mathrm{C}$ for $12 \mathrm{~h}$ to remove the pore solvents.

Preparation of Crystalline Ni-HAB (cNi-HAB). A solution of $\mathrm{Ni}\left(\mathrm{NO}_{3}\right)_{2} \cdot 6 \mathrm{H}_{2} \mathrm{O}(0.055$ mmol) and $0.4 \mathrm{~mL}$ of $14 \mathrm{M} \mathrm{NH}_{4} \mathrm{OH}$ in $2 \mathrm{~mL}$ of degassed $\mathrm{H}_{2} \mathrm{O}$ was added to a solution of $\mathrm{HAB} \cdot 3 \mathrm{HCl}(0.036 \mathrm{mmol})$ in $2 \mathrm{~mL}$ degassed $\mathrm{H}_{2} \mathrm{O}$ in a $20 \mathrm{~mL}$ scintillation vial. The vial 
was loosely capped and put under $65{ }^{\circ} \mathrm{C}$ for 2 hours without stirring. The product precipitated out as black solids. The mixture was centrifuged, decanted and washed with $5 \mathrm{~mL}$ deionized water for three times and then $5 \mathrm{~mL}$ methanol for another three times and then the solid product was kept in $3 \mathrm{~mL}$ methanol.

Fabrication of the sensory devices. The as-synthesized aNi-HAB sample was dispersed in deionized water to form a $0.5 \mathrm{mg} / \mathrm{ml}$ solution, then drop-casted onto a Si chip with pre-patterned $\mathrm{Ti}-\mathrm{Au}(20 / 50 \mathrm{~nm})$ electrodes to form a chemiresistive device (as illustrated in Figure S2c\&d). The sensory devices of crystal Ni-HAB were fabricated as above. The as-prepared sensory devices were treated at $80{ }^{\circ} \mathrm{C}$ on a hotplate for $10 \mathrm{~min}$ before each sensing test.

Chemical Sensing Measurement. Gas sensing measurements were conducted using a homemade system. The devices were placed in a sealed PDMS chamber to control the gas environment, and their electrical conductance was measured (Bias Voltage $=1 \mathrm{~V}$ ) on a test board by Keysight 2902a source-measure-unit (SMU). During the sensing tests, different concentrations of analyte gases were generated by mixing analyte gases (flowing the carrier gas (high purity dry $\mathrm{N}_{2}$ ) into a bubbler containing DI water, Acetone and Ethanol) with dry $\mathrm{N}_{2}$ at controlled ratio of gas flow rate and were passed through the gas chamber containing the sensor device.

General Characterizations. SEM image were recorded on Hitachi S-4800 with samples 
deposited on carbon conductive Tapes. SEM were obtained on JEOL JEM-1011 with samples deposited on carbon glue. XRD patterns were recorded on a Shimadzu Lab X/XRD-6000 X-ray diffractometer equipped with a $\mathrm{Cu}-\mathrm{K} \alpha$ radiation source $(\lambda=0.15418$ $\mathrm{nm}$ ) operating at $40 \mathrm{kV}$ and $30 \mathrm{~mA}$. FTIR spectra was acquired on a Bruker Vertex using a liquid nitrogen cooled detector. XPS was performed on a UIVAC-PHI 5000 Versa Probe spectrometer with $\mathrm{Al} \mathrm{K} \alpha$ as radiation source. Thermogravimetric analysis was performed on a TA Instruments at a heating rate of $3{ }^{\circ} \mathrm{C} / \mathrm{min}$ and a Nitrogen flow rate of $150 \mathrm{~mL} / \mathrm{min}$. BET was performed on a Micromeritics ASAP 2020 Surface Area and Porosity Analyzer. The sample was heated to $100{ }^{\circ} \mathrm{C}$, as determined by thermogravimetric analysis, under a dynamic vacuum of 4 mtorr until the outgas rate was less than 2 mtorr/minute. Mass spectra were recorded on a Bruker Apex IV Fourier Transform Ion Cyclotron Resonance. Liquid chromatography -mass spectrometry was performed on a UPLC-QTOT-MS, the chromatographic column is BEH C18 $(2.1 \times 50$ $\mathrm{mm}, 1.7 \mu \mathrm{m})$, the mobile phase is acetonitrile and water $(0.1 \% \mathrm{HCOOH})$, and the flow rate is $0.4 \mathrm{ml} / \mathrm{min}$. NMR uses the INOVA-400 MHz/600 MHz NMR spectrometer produced by Varian.

Theoretical calculation. All of the calculations were carried out using the Cambridge Serial Total Energy Package (CASTEP) $)^{1}$ module in the Materials Studio software package $^{2}$. The generalized gradient approximation (GGA) method together with the Perdew-Burke-Ernzerhof $(\mathrm{PBE})^{3}$ as exchange-correlation functional, and the ultrasoft 
pseudopotential was employed in both geometry optimizations and electronic property calculations. The Grimme method ${ }^{4-5}$ was applied to take the van der Waals interaction into consideration. To model the systems of cNi-HAB and aNi-HAB, a slab model in periodic boundary condition $(\mathrm{PBC})$ with the cell size of $26.8 \times 26.8 \times 20 \AA^{3}$ was employed. The vacuum thickness was large enough to avoid the interactions between the adjacent cells. The Brillouin zone (BZ) was sampled with a $1 \times 1 \times 1$ k-point for geometry optimization and a $3 \times 3 \times 1$ k-point for electronic structures. The energy convergence value between two consecutive steps was chosen as $2 \times 10^{-5} \mathrm{eV}$, and a maximum force was allowed lower than $0.05 \mathrm{eV} / \AA$ on each atom. The binding energy $\left(E_{b}\right)$ was obtained by calculating the energy difference between the total energy of the complex system $\left(E_{\text {complex }}\right)$ and the sum of individual energy of the Ni-HAB substrate ( $\left.\mathrm{E}_{\mathrm{Ni}-\mathrm{HAB}}\right)$ and $\mathrm{H}_{2} \mathrm{O}$ molecules $\left(\mathrm{E}_{\mathrm{H} 2 \mathrm{O}}\right)$, respectively, which was expressed as

$$
\mathrm{E}_{\mathrm{b}}=\mathrm{E}_{\text {complex }}-\mathrm{E}_{\mathrm{Ni}-\mathrm{HAB}}-\mathrm{E}_{\mathrm{H} 2 \mathrm{O}}
$$

The extent of charge transfer, $\delta_{e}$, was calculated by

$$
\delta_{e}=\mathrm{Q}_{\mathrm{Ni}-\mathrm{HAB}}-\mathrm{Q}_{\mathrm{H} 2 \mathrm{O}}
$$

where $\mathrm{Q}_{\mathrm{Ni}-\mathrm{HAB}}$, and $\mathrm{Q}_{\mathrm{H} 2 \mathrm{O}}$ represented the Mulliken charge populations of the Ni-HAB substrate, and the $\mathrm{H}_{2} \mathrm{O}$ molecules on the substrate, respectively. 


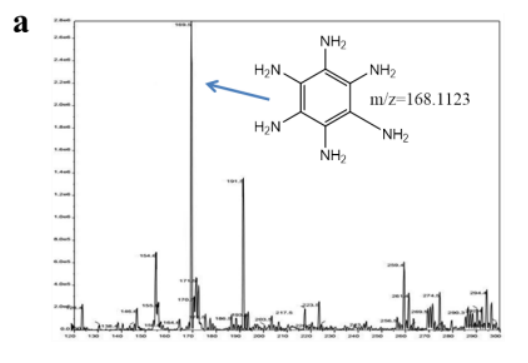

c

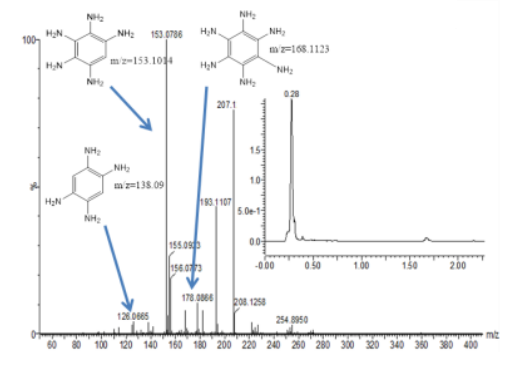

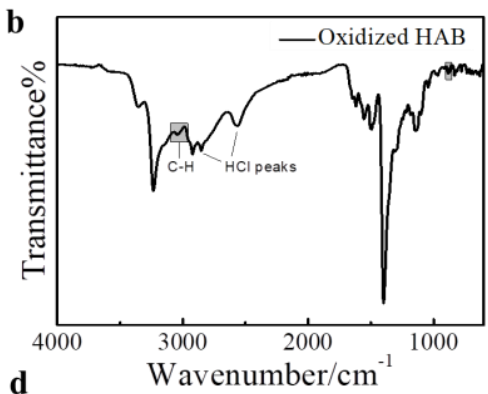

d

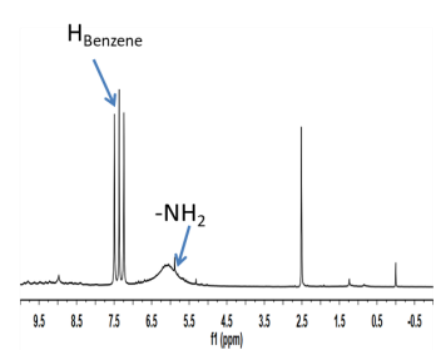

Figure S1. Characterization of oxidized and pure HAB. (a) MS spectrometry of pure HAB. (b-d) FT-IR (b), LC-MS (c) and NMR (d) results of oxidized HAB.

The multiple NMR peaks in benzene region (as well as broad $-\mathrm{NH}_{2}$ peak) and the different peaks in LC-MS corelated to different structures of oxidized HAB indicate that it is probably a mixture consisted of hexaaminobenzene, pentaaminobenzene, and tetraaminobenzne, where pentaaminobenzene is the major content. In addition, FT-IR of oxidized $\mathrm{HAB}$ showing $\mathrm{C}-\mathrm{H}$ vibration further supports the depletion of amino groups. For the convenience of further theoretical calculation and mechanistic investigation, pentaaminobenzene was selected as the ligand to propose the model of a defective aNi-HAB structure (that fits the final $\mathrm{Ni}$ to $\mathrm{N}$ ratio). 


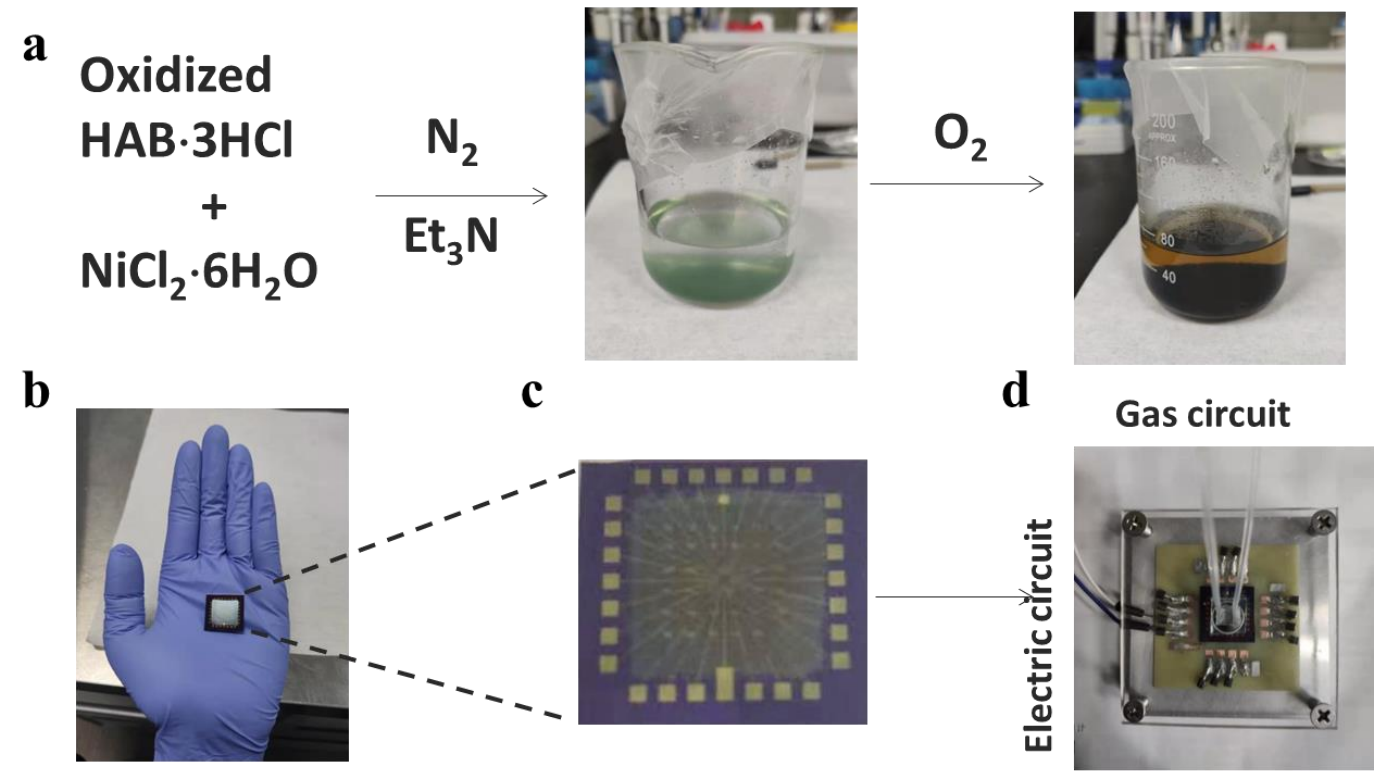

Figure S2. Synthesis of amorphous Ni-HAB and device fabrication. (a) Synthetic scheme of the missing-linker amorphous Ni-HAB by slow oxidation. (b) A photograph of an actual chemiresistor chip. (c) An optical image of the chip with micropatterned gold electrodes. (d) A photograph of the humidity sensor device. 

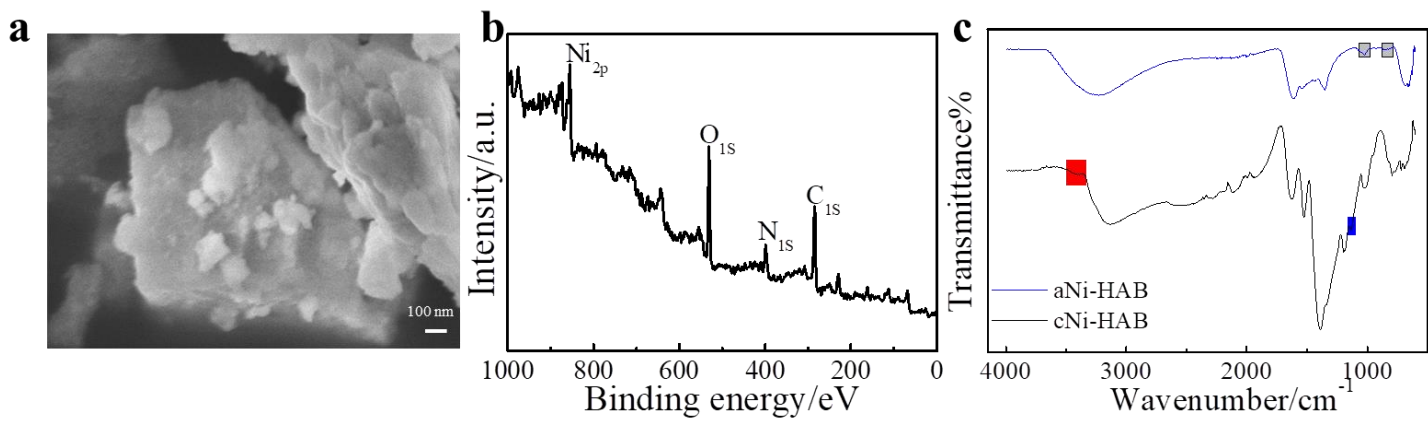

Figure S3. Characterizations of missing-linker aMOF. (a) Scanning electron microscopy (SEM) image of the missing-linker aMOF. (b) XPS of aMOF. (c) FT- IR of aNi-HAB and cNi-HAB. Gray mark in aNi-HAB at $860 \mathrm{~cm}^{-1}$ and $1022 \mathrm{~cm}^{-1}$ are attributed to the bending in-plane of unsubstituted $\mathrm{C}-\mathrm{H}$ in benzene. Blue mark in $1132 \mathrm{~cm}^{-1}$ belongs to quinoid ring. Rea mark at about $3220 \mathrm{~cm}^{-1}$ is the associative $\mathrm{N}-\mathrm{H}$ peak affected by water molecules.
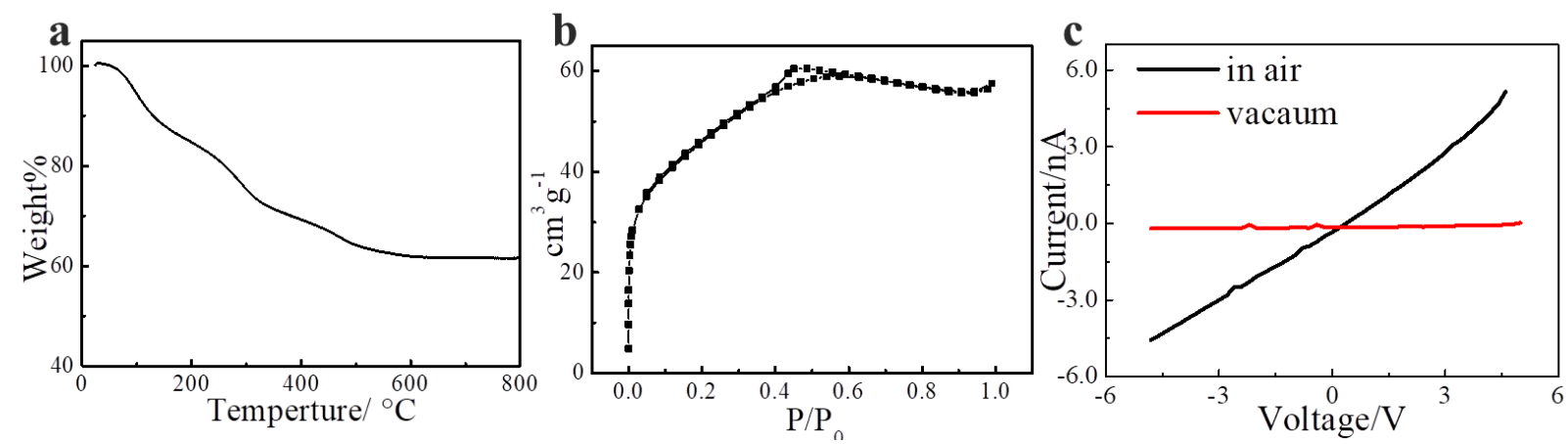

Figure S4. Characterization and I-V curves of amorphous Ni-HAB. (a)TGA image of aNi-HAB.(b) BET image of aNi-HAB.(c) I-V curves of aNi-HAB in air and vacuum. 


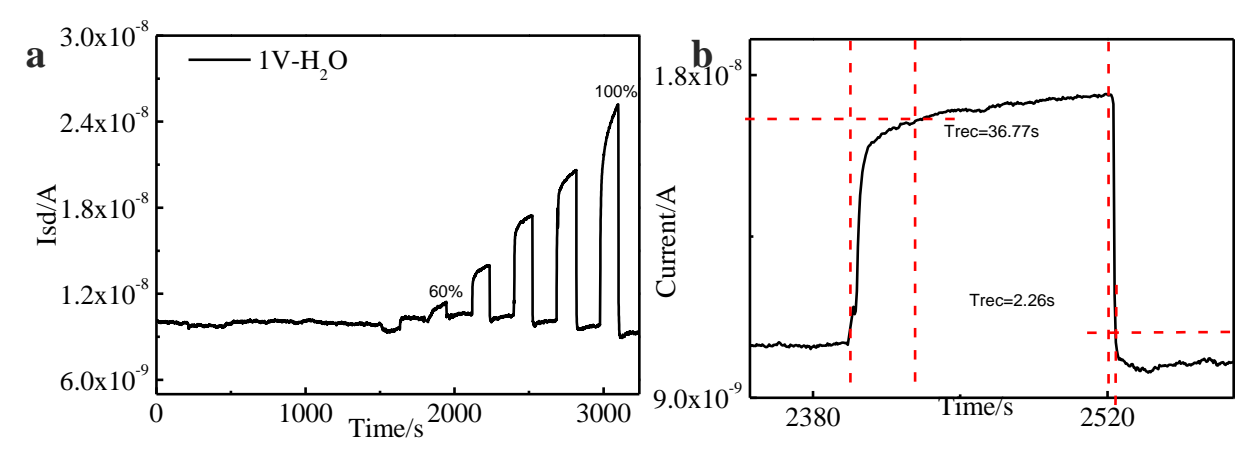

Figure S5. Humidity sensitivity of cNi-HAB devices. (a) Current response to dry air and different RH $(10 \%-100 \%)$ at room temperature; (b) response and recovery time at $80 \%$ RH.

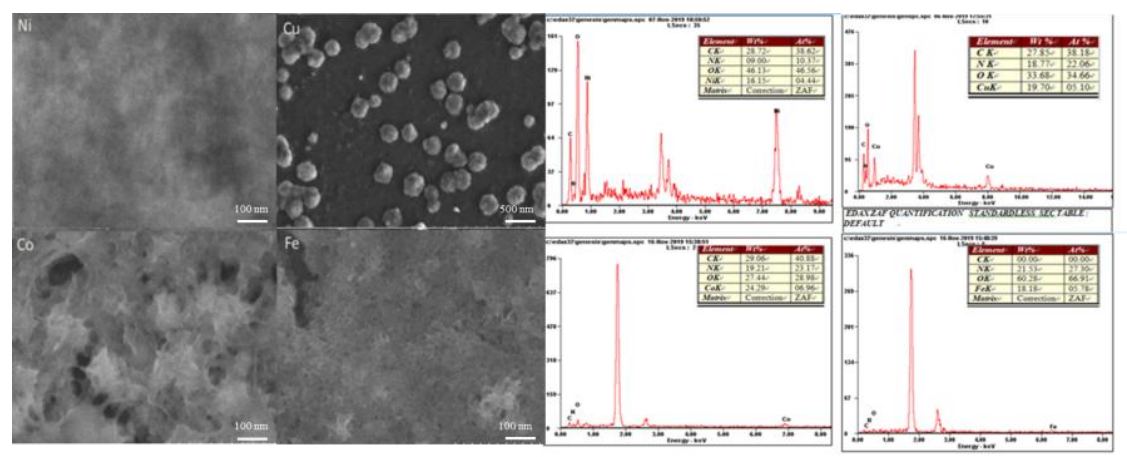

Figure S6. SEM image and EDS of aNi-HAB, aCu-HAB, aCo-HAB, aFe-HAB.

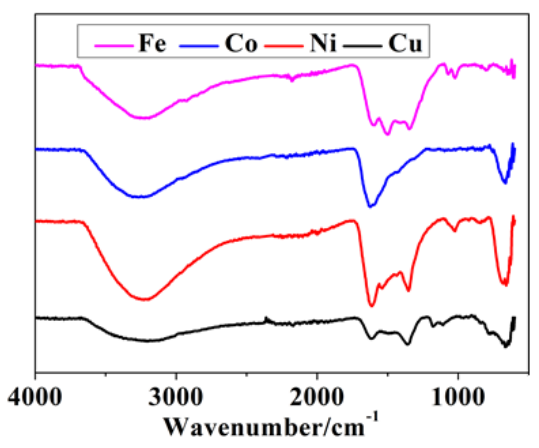

Figure S7. FTIR spectra of aNi-HAB, aCu-HAB, aCo-HAB, aFe-HAB. 

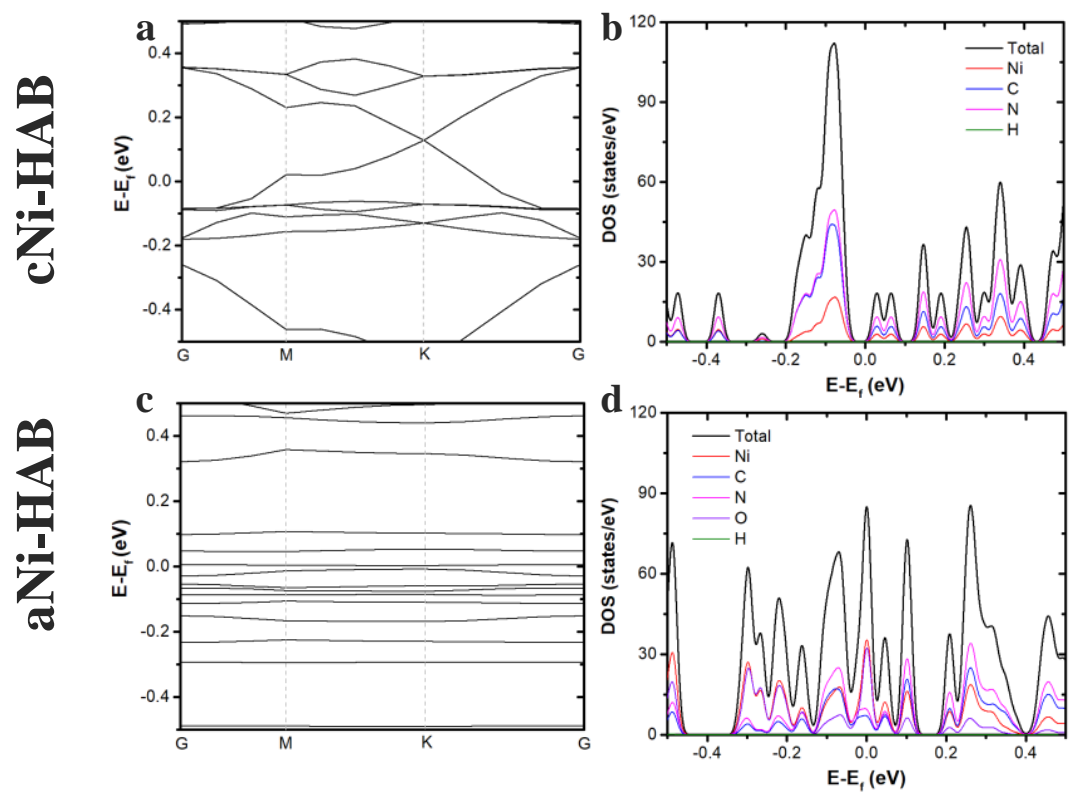

Figure S8. DFT calculated band structure. (a) and density of states (b) of cNi-HAB;

band structure (c) and density of states (d) of aNi-HAB, respectively.

\section{Reference}

1. M D Segall, P. J. D. L., M J Probert, C J Pickard1,PJ Hasni, S J Clark6 and M C Payne, First-principles simulation: ideas, illustrations and the CASTEP code. JOURNAL OF PHYSICS: CONDENSED MATTER 2002, 14, $2717-2744$

2. Materials Studio, version 7.0; Accelrys Inc.: San Diego, 2013

3. John P. Perdew, K. B., * Matthias Ernzerhof, Generalized Gradient Approximation Made Simple. Physical Review Letters 1996, 77, 3865-3868.

4. Grimme, S.; Antony, J.; Ehrlich, S.; Krieg, H., A consistent and accurate ab initio parametrization of density functional dispersion correction (DFT-D) for the 94 elements H-Pu. J Chem Phys. 2010, 132 (15), 154104.

5. Studio, M., Effect of the Damping Function in Dispersion Corrected Density Functional Theory. J Comput Chem 
2011, 32, 1456-1465. 